\title{
RU Cen and SX Cen: Two strongly depleted RV Tauri stars in binary systems ${ }^{\star}$
}

\section{The RV Tauri photometric b phenomenon and binarity}

\author{
T. Maas ${ }^{1}$, H. Van Winckel ${ }^{1, \star \star}$, and C. Waelkens ${ }^{1}$ \\ Instituut voor Sterrenkunde, K.U. Leuven, Celestijnenlaan 200B, 3001 Leuven, Belgium \\ Received 11 September 2001 / Accepted 6 February 2002

\begin{abstract}
We present a chemical abundance analysis on the basis of high signal-to-noise and high-resolution $(\lambda / \Delta \lambda \sim 48000)$ optical spectra of two RV Tauri stars RU Cen and SX Cen. With an $[\mathrm{Fe} / \mathrm{H}]=-1.9$ and a $[\mathrm{Zn} / \mathrm{Fe}]=+0.9$ for RU Cen and $\mathrm{a}[\mathrm{Fe} / \mathrm{H}]=-1.1$ and $\mathrm{a}[\mathrm{Zn} / \mathrm{Fe}]=+0.6$ for SX Cen, both stars of spectroscopic class B display strong depletion of refractory elements in their photospheres. Our CORALIE radial velocity measurements prove the stars to be members of binary systems and a detailed construction of the spectral energy distribution indicate the presence of a large amount of hot circumstellar dust. Moreover, the orbital period of SX Cen of around 600 days is similar to the published period of mean magnitude variation in the light curve (RV Tauri phomometric class $\mathrm{b}$ phenomenon). All these observations indicate the presence of a stable circumbinary disk in the objects and strengthen the model that this is a necessary condition for the depletion process to take
\end{abstract} \\ place.
}

Key words. stars: abundances - stars: AGB and post-AGB - stars: evolution - stars: individual: RU Cen stars: individual: SX Cen - stars: individual: RV Tauri

\section{Introduction}

The RV Tauri class of objects is a rather inhomogeneous group of luminous radially-pulsating stars which show a light curve with alternating deep and shallow minima. They were identified by Jura (1986) as Post-AGB objects on the basis of their high luminosity, their F, G and K spectral classes and the presence of circumstellar dust.

Preston et al. (1963) introduced a spectroscopic classification of RV Tauri stars: stars of type RVA are of spectral type G-K, show strong absorption lines but normal CN or $\mathrm{CH}$ bands while TiO bands sometimes appear at photometric minimum. RVB stars are generally somewhat hotter (spectral type $\mathrm{Fp}(\mathrm{R})$ ) weak lined objects which show enhanced $\mathrm{CN}$ and $\mathrm{CH}$ bands indicating evidence of enhanced carbon. RVC stars are also weak lined stars but show normal bands of $\mathrm{CH}$ and $\mathrm{CN}$. This last class contains the globular cluster RV Tauri stars. For historical reasons,

Send offprint requests to: T. Maas,

e-mail: Thomas.Maas@ster .kuleuven .ac.be

* Based on observations collected at the European Southern Observatory in Chile (64.L-0117(A), 67.D-0054(A)), on the $1.2 \mathrm{~m}$ Swiss Euler telescope on La Silla and on the $70 \mathrm{~cm}$ Swiss photometric telescope also on La Silla.

** Postdoctoral fellow of the Fund for Scientific Research, Flanders. also the photometric classes are labeled with "a" (which are the objects with a constant mean magnitude) and "b" (which are the objects with a long-term variability in the mean magnitude).

In recent years it became clear that RV Tauri photospheres often show chemical anomalies: elements with a high condensation temperature have low photospheric abundances, while elements with a low condensation temperature show higher abundances (Giridhar et al. 1994, 1998, 2000; Gonzalez et al. 1997a, 1997b; Van Winckel et al. 1998). There is general agreement that this is the result of gas-dust separation followed by selective reaccretion by the star of gas devoid of refractories.

The severity of this depletion abundance pattern differs from star to star but the general trend is that the spectroscopic class A objects are less or not affected by the gas-dust separation while the RVB stars are more severely affected. The studied stars of the spectroscopic class RVC, which have a low initial metallicity $([\mathrm{Fe} / \mathrm{H}] \leq-1.0)$, do not show any depletion (Giridhar et al. 2000).

The same depletion patterns, but much more extreme, were found in the binary Post-AGB stars HR 4049 (Lambert et al. 1988; Waelkens et al. 1991a) HD 52961 , (Waelkens et al. 1991b) and HD 44179, the central star of the Red Rectangle (Waelkens et al. 1992) and 
$\mathrm{BD}+39^{\circ} 4926$ (Kodaira et al. 1970). The metallicities of these stars are respectively $-4.8,-4.8,-3.4$ and -3.0 versus -2.1 which is the lowest metallicity of an RVB star (AD Aql) (Giridhar et al. 1998). Less extremely deficient Post-AGB stars are HD 46703 (Bond \& Luck 1987) and HD 213985 (Waelkens et al. 1991a). Radial velocity measurements proved that all these extremely deficient stars are binaries (Van Winckel et al. 1995) with orbital periods which surely prevented normal, single star AGB evolution. The objects therefore represent a peculiar stage in binary evolution. Waters et al. (1992) proposed a scenario which accounts for this depletion: in this scenario, the star will reaccrete clean gas from a stable circumbinary disc. For Post-AGB stars the presence of a circum-system disc implies binarity. This scenario was corroborated by the binarity of the extremely deficient Post-AGB stars and the detected dust discs around some of them (Waelkens et al. 1991a; Roddier et al. 1995; Bond et al. 1997).

Since the depletion patterns are observed in all field RV Tauri stars of type B and in some of type A, the binarity of all these stars should be addressed as well, together with the total absence of the depletion patterns in C-type RV Tauri stars studied so far. In the past, orbital elements have been determined for U Mon (Pollard \& Cottrel 1995), ACHer (Van Winckel et al. 1998) and EN Tra (Van Winckel et al. 1999). Orbital motion is also found for IW Car (Pollard et al. 1997) and EP Lyr (Gonzalez et al. 1997a). One of the reasons for this low number of known binaries among RV Tauri stars (122 members according to the fourth edition of the General Catalog of Variable Stars) is the fact that the detection of binarity for RV Tauri stars on the basis of radial velocity measurements is far from trivial. The presence of shocks in the atmosphere causes line-deformation and line-splitting which makes the determination of the radial velocity difficult. Moreover, to study the orbital motion, the radial velocity variations due to the pulsation have to be removed. This is hampered by the instability of the period and the velocity amplitude of the pulsation of most RV Tauri stars.

However, there are several indirect indications that the binary fraction among RV Tauri stars may be very high indeed (Van Winckel et al. 1999):

The spectral energy distributions of many RV Tauri stars show a considerable near IR-excess caused by a hot dust component (Gehrz 1972; Gehrz \& Ney 1972; Lloyd Evans 1985). This hot dust component is also present in the extremely depleted binary Post-AGB stars HR 4049, HD 44179 and HD 52961. In the latter binaries this hot dust emission is interpreted as coming from dust that is stored in a circumbinary disk close to the star, since there is no evidence for current dusty mass loss. The stability of the disk is further illustrated by the high level of dust processing in some of these discs as shown by the detailed analysis of mid-IR spectra (Molster et al. 1999).

Another argument comes from the weak circumstellar CO-emission of RV Tauri stars. The CO-emission and the flux at $60 \mu \mathrm{m}$ in single Post-AGB stars show the same correlation as is observed in AGB-stars. In the objects with
Table 1. Basic parameters of RU Cen and SX Cen. The parameters are from SIMBAD except for the periods (for RUCen Pollard et al. 1996 and for SX Cen Kholopov et al. 1999) and the mean visual magnitude and the visual amplitude for RU Cen, Pollard et al. (1996).

\begin{tabular}{|c|c|c|c|}
\hline & & RUCen & SX Cen \\
\hline HD-number & & HD 105578 & HD 107439 \\
\hline \multirow[t]{2}{*}{ Coordinates } & $\alpha_{2000}$ & 120923.8 & 122112.6 \\
\hline & $\delta_{2000}$ & -452534.8 & -491241.1 \\
\hline Mean $m_{\mathrm{v}}$ & & 9.05 & 9.03 \\
\hline Amplitude $m_{\mathrm{V}}$ & & 1.28 & \\
\hline Period(days) & & 64.6 & 32.8642 \\
\hline Galactic & 1 & 295.25 & 297.87 \\
\hline coordinates & $\mathrm{b}$ & +16.82 & +13.36 \\
\hline Parallax(mas) & & .61 & \\
\hline
\end{tabular}

an expanding dust shell, there is no big spread in gas-todust ratio. The RV Tauri stars do not follow this relation and are deficient in CO. For other objects which show weak and narrow circumstellar CO, a stable dusty disc is suspected (Jura \& Kahane 1999 and references therein).

Clearly, to directly reveal the binary nature of an RV Tau star, detailed monitoring is needed over many photometric cycles. In this paper we report upon such a monitoring programme and discuss two RV Tauri stars of spectroscopic type B: RU Cen and SX Cen. Both stars are known to be rather regular pulsators. RUCen is of the photometric class a, while SX Cen is of the photometric class $b$, with a period of the long time variation in mean magnitude of 615 days (Lloyd Evans 1985 quoted from O'Connell 1933 and Voûte 1940). The basic parameters of these stars are given in Table 1 . The outline of this paper is as follows: in Sect. 2 we give an overview of the accumulated observations. In the third section we show that both stars show a strong depletion in the photospheric abundances. In the fourth section we construct and discuss the spectral energy distribution. In Sect. 5 we treat the CORALIE radial velocities. We also used the CORALIE spectra to study the changes in the $\mathrm{H} \alpha$ line profile during the different phases of the pulsation cycle. In Sect. 6 we discuss all observed data of the two stars together, and we summarize our main conclusions.

\section{Observations}

\subsection{Spectroscopy}

\subsubsection{FEROS spectra}

High-resolution, high signal-to-noise spectra for RU Cen and SX Cen were obtained with the fiber-fed spectograph FEROS mounted on the ESO 1.52-m telescope at la Silla. These spectra ranging from $3600 \AA$ to $8900 \AA$ are obtained in one integration on a monolithic $2 \mathrm{~K} \times 4 \mathrm{~K} \mathrm{EEV}$ CCD chip. The log of the observations is given in Table 2 together with the obtained signal-to-noise ratio in the red. An echelle spectrum does not have a very homogeneous 
Table 2. The log of the FEROS spectra and the signal-to-noise around $5850 \AA$.

\begin{tabular}{|c|c|c|}
\hline & Date and UT & $S / N$ \\
\hline RU Cen & $24 / 03 / 002: 51$ & $250-350$ \\
\hline SX Cen & 24/03/00 3:40 & $100-150$ \\
\hline SX Cen & 27/06/01 0:01 & \\
\hline SX Cen & 27/06/01 1:03 & $200-250$ \\
\hline
\end{tabular}

$S / N$ so we list the range of $S / N$ obtained near the blaze wavelengths of different spectral orders. The standard reduction was performed by the pipe-line reduction in the MIDAS environment, and included bias subtraction, wavelength calibration (using a Th-Ar lamp comparison spectrum) and flat-fielding after extraction. The extraction was performed as a simple average of the extracted pixels with cosmic hit clipping. The two spectra of SX Cen taken on June 27th 2001 were added. We normalised the spectra interactively using smoothed splines. Sample spectra are shown in Figs. 4-7 and 12.

\subsubsection{Coralie data}

We have been observing RU Cen and SX Cen since June 2000 with the spectrograph CORALIE mounted on the $1.2 \mathrm{~m}$ Euler Swiss Telescope at La Silla, ESO Chile. CORALIE (Queloz et al. 1999), with which several extrasolar planets were detected recently, is an improved version of the ELODIE (Baranne et al. 1996) spectrograph. CORALIE has a very stable configuration and is fed by an optical fibre with an aperture projected on the sky of $1^{\prime \prime}$. We used the on-line data-reduction and crosscorrelation system. Until now we gathered for RU Cen 48 and for SX Cen 46 low signal-to-noise but high resolution $(\lambda / \delta \lambda=50000)$ spectra and we will enlarge this sample in the future. The radial velocities are computed using cross-correlation with a software template. For RU Cen we used a template of spectral type F, for SX Cen a template of spectral type G. We also used two old measurements from the CORAVEL spectrograph mounted on the Danish $1.5 \mathrm{~m}$ ESO telescope.

\subsection{Photometry}

Our optical photometric measurements of the two objects were obtained with the $70 \mathrm{~cm}$ Swiss telescope at La Silla using the Geneva 7-band photometric system. These measurements are, however, too sparse (26 for RU Cen and 19 for SX Cen) to study the photometric behaviour of both objects in detail. Therefore, we only used these data to construct a spectral energy distribution (SED) (see following section). We obtained near-IR photometry with the ESO JHKLM bands with the ESO $1 \mathrm{~m}$ telescope at La Silla for RUCen and with the $2.2 \mathrm{~m}$ ESO telescope for SX Cen.

\section{Spectral energy distribution}

The main problem in constructing an SED of pulsating stars is the difficulty of obtaining simultaneous data in a wide spectral domain. We therefore constructed the SEDs with (spectro) photometric data of the same pulsation phase but obtained during different cycles.

For RU Cen the UV data, which were downloaded from the newly extracted IUE archive, were obtained at pulsation phase $0.37(15 / 02 / 1995)$ using the ephemeris given in Sect. 5, while for SX Cen we used the IUE spectra obtained around pulsation phase 0.85 (08/05/1995) using the ephemeris also given in Sect. 5. The optical and near-IR photometry of both stars was selected at the same pulsation phase while for the far-IR fluxes, where variations with phase do not occur, we used the IRAS point-source catalogue.

The total reddening was determined by minimizing the difference between the dereddened fluxes in the optical and the Kurucz model atmosphere with the correct metallicity. For the model parameters $T_{\text {eff }}, \log g$ and the overall metallicity, we used the values obtained during our chemical analysis study of both objects. The SED are given in Figs. 1 and 2. For RU Cen we obtained a total reddening of $E(B-V)=0.6 \pm 0.1$ while for SX Cen a total reddening of $E(B-V)=0.3 \pm 0.1$ was found. Note that, unfortunately, the parameters of the Kurucz models were obtained using spectroscopic data at another pulsation phase. To test the influence on the reddening estimate, we used the same photospheric data and fitted a $500 \mathrm{~K}$ cooler model through the points. The best fit was obtained for a total reddening of $E(B-V)=0.4$, while the main conclusions concerning the dust-excess remain the same.

The main difference between the SEDs of both objects and the ones of post-AGB stars of similar effective temperature with a detached expanding circumstellar shell is the presence of a strong near-IR excess. RU Cen is known to be a RV Tauri star with a relatively modest near-IR excess (see e.g. Lloyd Evans 1999) but the SED indicates that the IR excess does start around the $L$ band $(3.8 \mu \mathrm{m})$. The SED of SX Cen was already extensively studied by Shenton et al. (1994) and we confirm their findings that there is good evidence for an IR-excess starting already at $K(2.2 \mu \mathrm{m})$. Assuming a normal expansion velocity, they argue that the hot dust component indicates that SX Cen left the AGB decades ago or that it is in the process of leaving the AGB at this very moment.

Another alternative explanation is advocated here, based on the comparison between the SEDs and these of the post-AGB stars with near-IR excesses in their SED. In previous monitoring campaigns, it became clear that post-AGB candidates with a near IR-excess and with a low amplitude photometric variability (for which binary motion is more easily detectable), turned out to be binaries (e.g. Waters et al. 1997; Van Winckel 2001 and references therein). In these papers, the presence of a near-IR excess in post-AGB stars is taken to be a signature of a dusty disc, not of an outflow. 


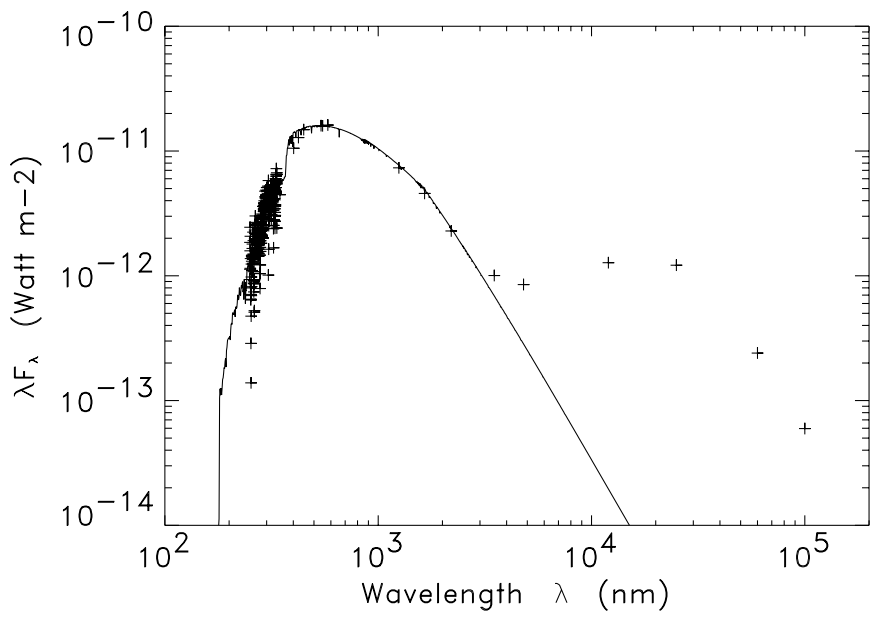

Fig. 1. The SED of RUCen. The photometric data (+ signs) are from the same pulsation phase, the full line is the appropriate Kurucz-model. We used a total reddening of $E(B-V)=0.6$.

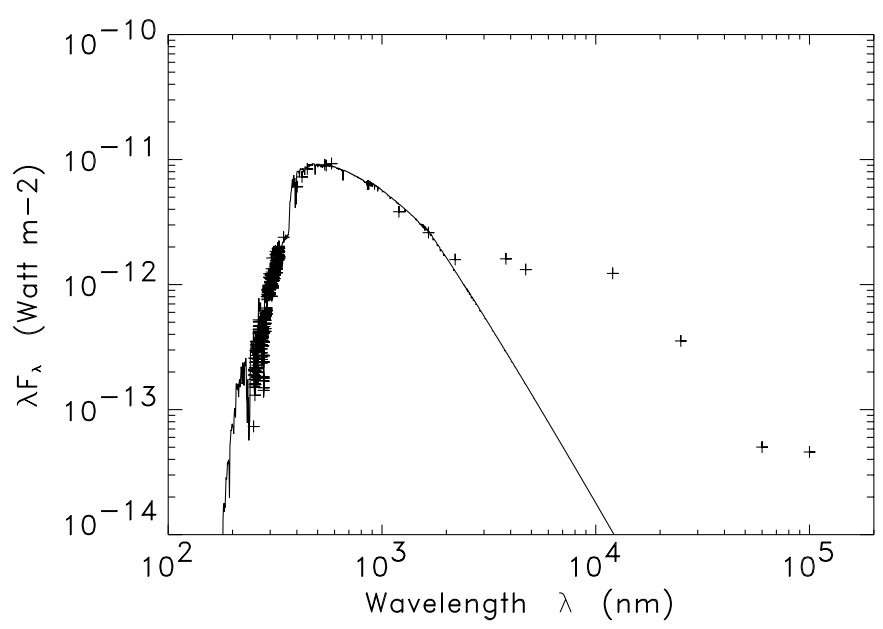

Fig. 2. The SED of SX Cen. Same symbols as in Fig. 1. We obtained a total reddening of $E(B-V)=0.3$. Note that the $100 \mu \mathrm{m}$ IRAS fluxpoint is an upper limit.

\section{Chemical analysis}

\subsection{Atmospheric parameters}

The abundances are calculated on the basis of a LTE model atmospheres of Kurucz (1993) and the LTE program MOOG of Prof. C. Sneden. A model atmosphere is determined by its effective temperature $\left(T_{\text {eff }}\right)$, the gravity $(\log g)$ and the metallicity $([\mathrm{Fe} / \mathrm{H}])$. We determined these parameters on the basis of spectroscopic criteria: the effective temperature by forcing the abundances of Fe I-lines to be independent of the excitation potentials and the gravity by forcing ionsiation balance between the Fe I and Fe II lines. Moreover, the microturbulent velocity was estimated by forcing the abundance of the Fe I-lines to be independent of the reduced equivalent width. We then used the model atmospheres with the correct overall metallicity as given by the Fe abundance.
The oscillator strengths of the lines were taken from the critically compiled database continuously updated in our institute. For more details we refer to Van Winckel \& Reyniers (2000).

As we will show in Sect. 5.1.1, RU Cen was observed at a pulsation phase at which parts of the line-forming atmosphere were perturbed by shocks. This resulted, for some transitions, in a splitting of the line. The Fe I-lines and FeII-lines of RU Cen which showed this splitting have on average a rather high equivalent width (EW hereafter) since these lines are formed higher in the atmosphere. We excluded these lines for the determination of the model parameters. In this way the remaining list consisted of 48 FeI-lines and 16 FeII-lines. The EW-determination was determined by single Gaussian fitting.

For RUCen we obtained $T_{\text {eff }}=6000 \mathrm{~K}, \log g=1.5$ with a typical error of $\pm 250 \mathrm{~K}$ and 0.5 respectively and $[\mathrm{Fe} / \mathrm{H}]=-2.0$. For the spectrum of SX Cen taken on April 242000 (first spectrum) we obtained $T_{\text {eff }}=6000 \mathrm{~K}$, $\log g=1.0$ and $[\mathrm{Fe} / \mathrm{H}]=-1.0$ and for that taken on June 272001 (second spectrum) $T_{\text {eff }}=6250 \mathrm{~K}, \log g=1.5$ and $[\mathrm{Fe} / \mathrm{H}]=-1.0$.

\subsection{Abundances of RU Cen}

The abundance results obtained for RU Cen are listed in Table 3. In Fig. 3 the abundance of the elements are plotted versus their dust condensation temperature.

We adopted very strong line-selection criteria in our analysis of RU Cen using only lines with clear profiles and an equivalenth width smaller than $100 \mathrm{~m} \AA$ and in most cases even smaller than $50 \mathrm{~m} \AA$. Thanks to our very high $S / N$ between 200 and 250 (see Table 2) we could measure a fair amount of undisturbed small lines (143 in total) for our chemical analysis. In Table 3 we list also the mean equivalenth width of the lines used for every ion. We carefully compared the spectrum of RUCen with the one of $\mathrm{ACHer}$ to check for blending and splitting. ACHer is a good comparison star since it has similar model parameters but is less depleted and shows narrow but clearly systematically stronger lines. The small line-to-line scatter of the abundances of ions for which we obtained a fair amount of lines (Col. $\sigma$ ) illustrate that stringent lineselection criteria do yield consistent results, even if the stronger lines in the spectrum show line splitting and an upper atmosphere not in hydrostatic equilibrium. Due to the strong depletion only $\mathrm{Cr}$ shows small lines of a different ionisation stage. The difference in abundance of only 0.1 dex is a good consistency check on the model parameters.

Abundances crucial to determine whether the chemical composition is due to depletion or not, are those of $\mathrm{Zn}$ and $\mathrm{S} . \mathrm{Zn}$ is an element with a nucleosynthesis very similar to that of $\mathrm{Fe}$, but with a much lower condensation temperature. The abundance of $\mathrm{Zn}[\mathrm{Zn} / \mathrm{H}]=-1.0$ or $[\mathrm{Zn} / \mathrm{Fe}]=+0.9$ undoubtedly points in the direction of a depletion process. This abundance is well established 
Table 3. Chemical analysis of RU Cen. The solar abundances to compute the $[\mathrm{el} / \mathrm{H}]$ ratios are taken from Anders \& Grevesse (1989). The dust condensation temperatures are from Lodders and Fegley (1988) and references therein. They are computed using a solar abundance mix at a pressure of $10^{-4}$ atm. They do not ascribe a condensation temperature to $\mathrm{O}$ as it is the most abundant element in rock and therefore a separate condensation temperature is meaning less.

\begin{tabular}{|c|c|c|c|c|c|c|}
\hline \multicolumn{7}{|c|}{$\begin{array}{c}\text { RU Cen } \\
T_{\text {eff }}=6000 \mathrm{~K} \\
\log g=1.5 \\
\xi_{\mathrm{t}}=3.50 \mathrm{~km} \mathrm{~s}^{-1} \\
{[\mathrm{Fe} / \mathrm{H}]=-2.0}\end{array}$} \\
\hline ion & $N$ & $\overline{W_{\lambda}}$ & $\epsilon$ & $\sigma$ & {$[\mathrm{el} / \mathrm{H}]$} & $T_{\text {cond }}$ \\
\hline C I & 10 & 42 & 8.14 & 0.12 & -0.42 & 78 \\
\hline N I & 2 & 10 & 7.72 & 0.03 & -0.33 & 120 \\
\hline O I & 4 & 11 & 8.57 & 0.08 & -0.36 & \\
\hline $\mathrm{Na} \mathrm{I}$ & 2 & 25 & 5.19 & 0.08 & -1.14 & 970 \\
\hline Mg I & 2 & 42 & 5.90 & 0.06 & -1.68 & 1340 \\
\hline S I & 6 & 14 & 6.53 & 0.10 & -0.68 & 674 \\
\hline $\mathrm{Ca} \mathrm{I}$ & 7 & 30 & 4.47 & 0.06 & -1.89 & 1634 \\
\hline Sc II & 1 & 21 & 1.22 & & -1.88 & 1652 \\
\hline Ti II & 8 & 24 & 3.03 & 0.15 & -1.96 & 1600 \\
\hline V II & 2 & 46 & 2.23 & 0.04 & -1.77 & 1455 \\
\hline Cr I & 4 & 18 & 3.75 & 0.08 & -1.92 & 1301 \\
\hline Cr II & 2 & 15 & 3.85 & 0.18 & -1.82 & \\
\hline Mn I & 3 & 33 & 3.75 & 0.14 & -1.64 & 1190 \\
\hline $\mathrm{Fe} I$ & 66 & 32 & 5.65 & 0.12 & -1.87 & 1337 \\
\hline $\mathrm{Fe} I I$ & 16 & 40 & 5.60 & 0.10 & -1.92 & \\
\hline Co I & 1 & 37 & 3.09 & & -1.83 & \\
\hline Ni I & 5 & 17 & 4.57 & 0.10 & -1.68 & 1354 \\
\hline Zn I & 2 & 54 & 3.60 & 0.03 & -1.00 & 684 \\
\hline Zr II & 1 & 19 & 0.95 & & -1.65 & 1717 \\
\hline La II & & & -0.23 & & $<-1.45$ & 1520 \\
\hline Ce II & & & -0.43 & & $<-1.98$ & 1599 \\
\hline Pr II & & & -0.78 & & $<-1.49$ & 1532 \\
\hline Nd II & & & -0.64 & & $<-2.14$ & 1563 \\
\hline Sm II & & & -0.67 & & $<-1.67$ & 1515 \\
\hline
\end{tabular}

by two lines with nice profiles which results in a high internal accuracy $\sigma=0.03$ (see Figs. 5 and 6 ). $\mathrm{S}$ is an $\alpha$ element with a lower condensation temperature than the other $\alpha$-elements $\mathrm{Mg}, \mathrm{Si}, \mathrm{Ca}$ and $\mathrm{Ti}$. The abundance of $\mathrm{S}[\mathrm{S} / \mathrm{Fe}]=+1.2$ versus an average abundance $[\alpha / \mathrm{Fe}]=$ +0.2 of $\mathrm{Mg}, \mathrm{Si}, \mathrm{Ca}$ and $\mathrm{Ti}$ confirms the depleted nature of the photosphere of RU Cen. Note that the $[\mathrm{Zn} / \mathrm{Fe}]$ and $[\mathrm{S} / \mathrm{Fe}]$ abundance ratios are much less dependent on the model atmosphere parameters than the values of the absolute abundances. By increasing the model temerature by as much as $500 \mathrm{~K}$, one obtaines a difference in Fe I abundance of 0.26 dex. This is similar to the Zn I abundance difference of 0.23 making the $[\mathrm{Zn} \mathrm{I} / \mathrm{Fe} \mathrm{I}]$ abundance of this hotter model nearly equal than for our preferred model. The relative abundance ratios are therefore very

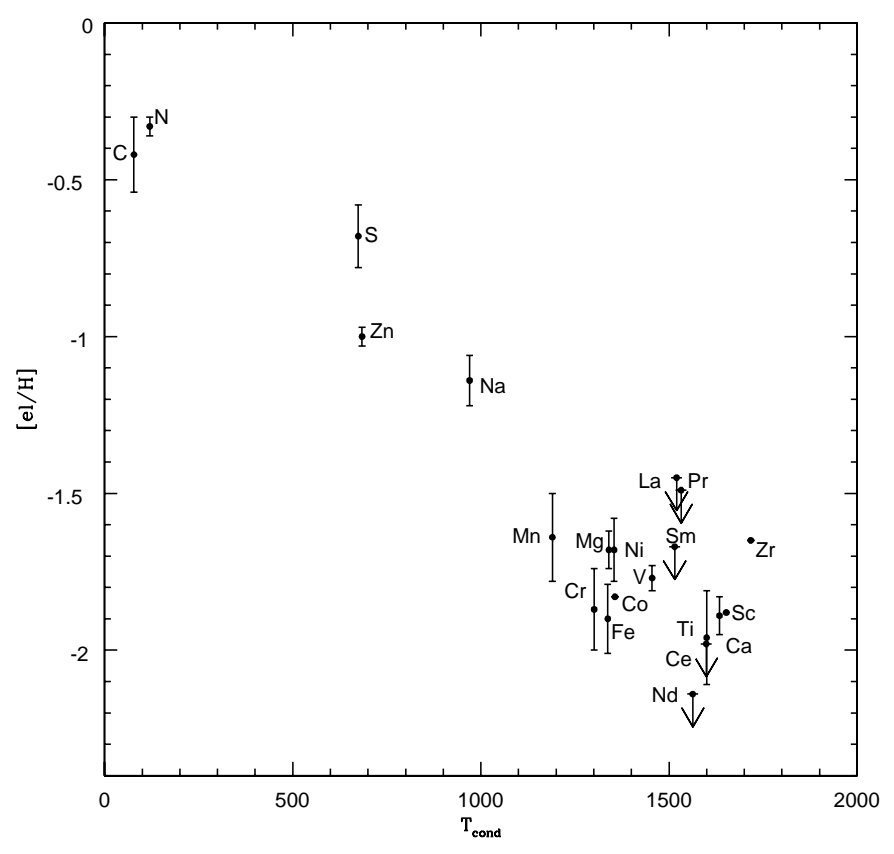

Fig. 3. The composition of RU Cen against the dust condensation temperature of the chemical element.

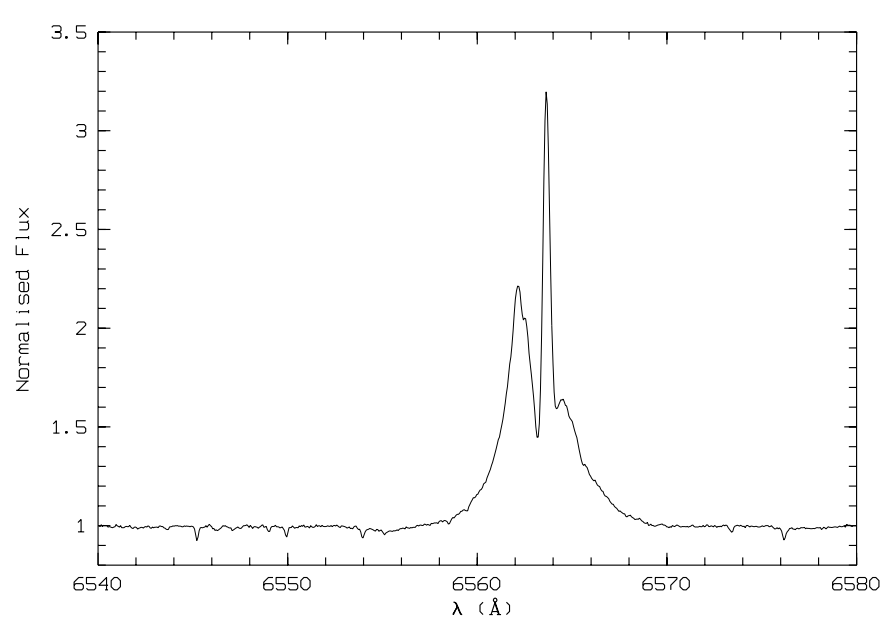

Fig. 4. The normalised FEROS spectrum of RU Cen around $\mathrm{H} \alpha$.

robuust against errors in the model parameters. Although the spectrum of RUCen was not obtained during an optimal pulsation phase, all the abundances yield a consistent picture (see Fig. 3) with a clear correlation between the underabundance of the chemical element and its condensation temperature. Since all studied RV Tauri stars of spectral type RVB show this depletion pattern, it does not come as a surprise. RU Cen belongs to the more depleted of the sample studied till now (see Table 8 of Giridhar et al. 2000).

The documented carbon abundance of $[\mathrm{C} / \mathrm{Fe}]=+1.5$ does not reflect the internal nucleosynthesis history of the object. For suspected post-AGB stars one might expect to find an enrichment of carbon and also of $s$-process elements, but due to the depletion process, the $\mathrm{C} / \mathrm{Fe}$ 


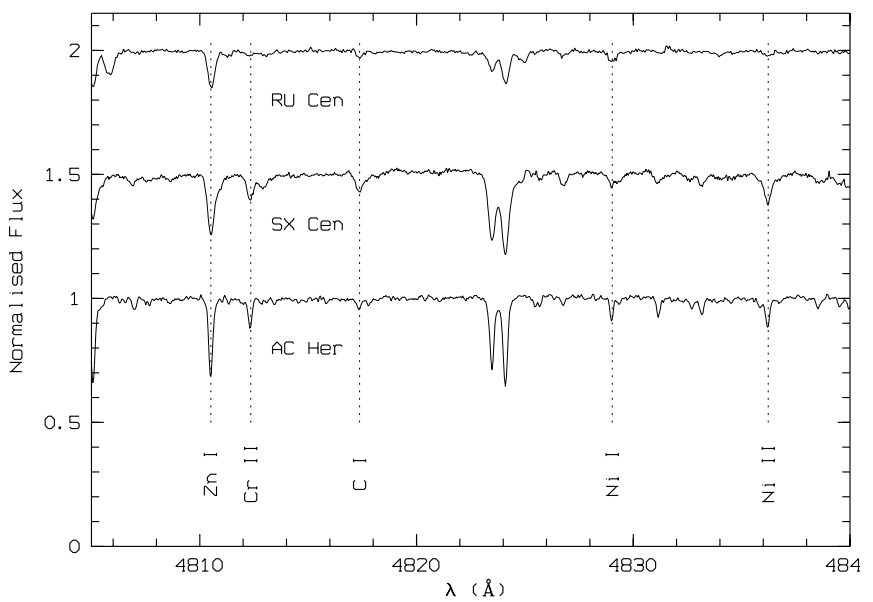

Fig. 5. Sample spectra of SX Cen and RU Cen around the Znline at $4810.54 \AA$. The spectra are velocity corrected. At the bottom the depleted RV Tauri star AC Her (Van Winckel et al. 1998) is plotted.

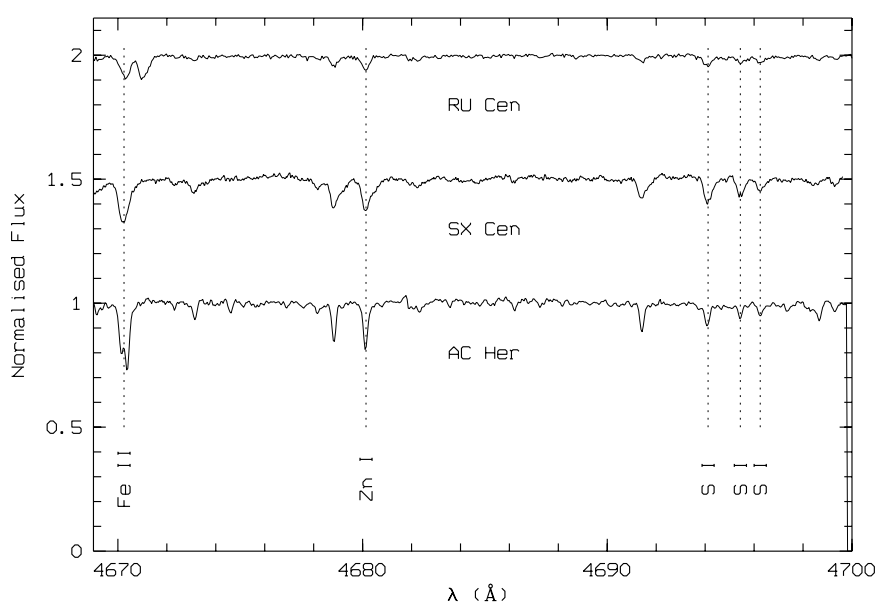

Fig. 6. Sample spectra of SX Cen and RU Cen around the Znline at $4680.140 \AA$ and the $\mathrm{S}$ triplet around $4695 \AA$. The spectra are velocity corrected. At the bottom the depleted RV Tauri star AC Her (Van Winckel et al. 1998) is plotted.

ratio is heavily affected. $s$-process elements show, on general, a high dust condensation temperature so their abundances follow the depletion of Fe. Only $\mathrm{Zr}$ shows a line-profile which is consistent with our selection criteria and we found a slight overabundance of $[\mathrm{Zr} / \mathrm{Fe}]=$ +0.3 , although it has a higher condensation temperature than Fe. The $N$-abundance is based on only two very small lines. The stronger $N$-lines around $8685 \AA$ are present but were not used because the line-profiles were affected by artificial signal (cosmic hits, spectral order matching problems). For the other s-process elements (La, Ce, Pr, Nd and $\mathrm{Sm}$ ) we derived an upper limit for the abundance since even the strongest expected lines were not present in the spectra. The upper limit is therefore derived assuming a fixed EW of $5 \mathrm{~m} \AA$ for the strongest line in a clear part of the spectrum. We used the line lists of the Vienna Atomic Line Database (Vald2,

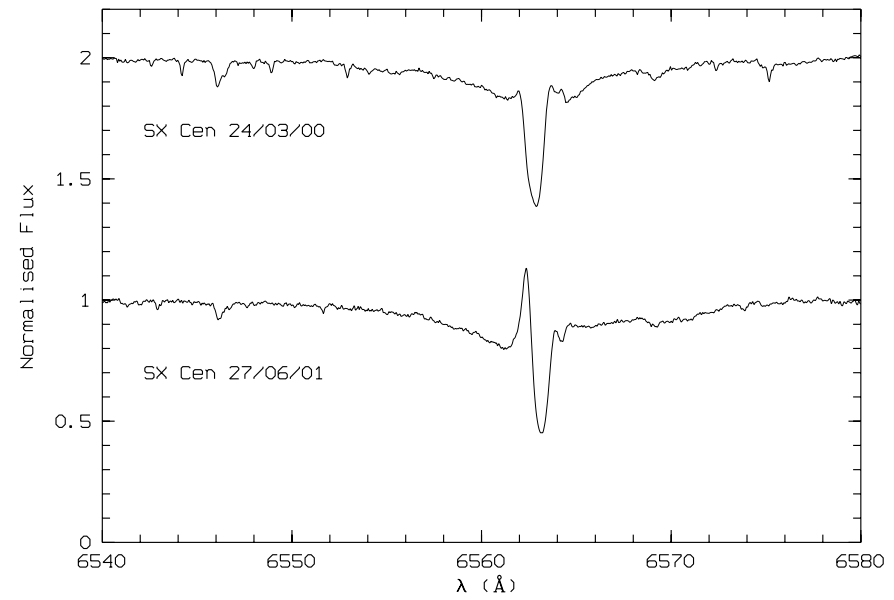

Fig. 7. The normalised FEROS spectrum of SX Cen around $\mathrm{H} \alpha$.

http://www.astro.univie.ac.at/vald/, Kupka et al. 1999) to look for these strongest optical lines. From the $s$-process/Fe ratio, one can conclude that there is no evidence for AGB chemical enrichment in RU Cen.

Luck \& Bond (1989) carried out a chemical abundance analysis of RUCen on the basis of photographic spectra. They derived also a metal deficiency with $[\mathrm{Fe} / \mathrm{H}]=-1.4$ but didn't determine abundances for $\mathrm{S}$ and $\mathrm{Zn}$ which made the link to the depletion history of the object not obvious. For the common elements there is an agreement for $\mathrm{O}, \mathrm{Na}$, $\mathrm{Mg}$, Si, Ca, Ti, Mn, Fe, Ni. For the elements Sc, V, Cr, $\mathrm{Ba}, \mathrm{Nd}$ the differences are larger.

To summarise, the correlation between the photospheric underabundances and the condensation temperature is clear: RU Cen is an RV Tauri where the depletion process has altered the photospheric abundances severely $([\mathrm{Fe} / \mathrm{H}]=-1.9,[\mathrm{~S} / \mathrm{Fe}]=+1.2,[\mathrm{Zn} / \mathrm{Fe}]=+0.9)$.

\section{3. $S X$ Cen}

Since the star is strongly variable, we derived the atmospheric parameters and computed the chemical composition based on the two spectral measurements independently. The relative abundances were consistent. We list here the results of the second spectrum because of its higher signal to noise, less deformed line profiles and more accurate abundances for $\mathrm{N}$ and $\mathrm{O}$ based on more detected lines. The results are listed in Table 4 while in Fig. 8 we show the abundance of the elements versus their condensation temperature.

The low Fe-abundance $([\mathrm{Fe} / \mathrm{H}]=-1.1)$ combined with the high abundance of $\mathrm{Zn}([\mathrm{Zn} / \mathrm{H}]=-0.5$ or $[\mathrm{Zn} / \mathrm{Fe}]=$ $+0.6)$ and of $\mathrm{S}([\mathrm{S} / \mathrm{H}]=-0.1$ or $[\mathrm{S} / \alpha]=+1.2$ with $\alpha=\mathrm{Mg}, \mathrm{Si}, \mathrm{Ca}$ and $\mathrm{Ti}$ ) prove that the photospheric abundances of SX Cen are determined by depletion rather than internal nucleosynthesis and dredge-up processes (see Figs. 5 and 6 ). Also here the Fe abundance is not a reliable measure of the initial metallicity. The correlation between the condensation temperature and the photospheric 
Table 4. Same as Table 3 but now for SX Cen.

\begin{tabular}{|c|c|c|c|c|c|c|}
\hline \multicolumn{7}{|c|}{$\begin{array}{c}\text { SX Cen } \\
T_{\text {eff }}=6500 \mathrm{~K} \\
\log g=1.5 \\
\xi_{\mathrm{t}}=4 \mathrm{~km} \mathrm{~s}^{-1} \\
{[\mathrm{Fe} / \mathrm{H}]=-1.0}\end{array}$} \\
\hline ion & $N$ & $\overline{W_{\lambda}}$ & $\epsilon$ & $\sigma$ & {$[\mathrm{el} / \mathrm{H}]$} & $T_{\text {cond }}$ \\
\hline C I & 14 & 74 & 8.50 & 0.10 & -0.06 & 78 \\
\hline N I & 3 & 93 & 8.42 & 0.05 & +0.37 & 120 \\
\hline O I & 3 & 46 & 9.00 & 0.15 & +0.07 & \\
\hline $\mathrm{Na} \mathrm{I}$ & 3 & 71 & 6.29 & 0.14 & -0.04 & 970 \\
\hline Mg I & 1 & 33 & 6.61 & & -0.97 & 1340 \\
\hline Si II & 2 & 98 & 6.77 & 0.05 & -0.78 & 1529 \\
\hline S I & 6 & 54 & 7.13 & 0.13 & -0.08 & 674 \\
\hline $\mathrm{Ca} \mathrm{I}$ & 4 & 75 & 4.84 & 0.11 & -1.52 & 1634 \\
\hline Sc II & 2 & 50 & 1.14 & 0.11 & -1.96 & 1652 \\
\hline Ti II & 4 & 37 & 3.02 & 0.08 & -1.97 & 1600 \\
\hline Cr I & 3 & 56 & 4.45 & 0.10 & -1.22 & 1301 \\
\hline Cr II & 8 & 61 & 4.68 & 0.12 & -0.99 & \\
\hline Mn I & 2 & 65 & 4.60 & 0.06 & -0.79 & 1190 \\
\hline $\mathrm{Fe} I$ & 48 & 63 & 6.36 & 0.13 & -1.16 & 1337 \\
\hline Fe II & 18 & 64 & 6.38 & 0.14 & -1.14 & \\
\hline $\mathrm{Ni} \mathrm{I}$ & 1 & 23 & 4.95 & & -1.30 & 1354 \\
\hline $\mathrm{Cu} \mathrm{I}$ & 1 & 62 & 3.99 & & -0.22 & 1170 \\
\hline Zn I & 2 & 93 & 4.06 & 0.07 & -0.54 & 684 \\
\hline Y II & 2 & 82 & 1.27 & 0.07 & -0.97 & 1622 \\
\hline Zr II & 1 & 37 & 1.37 & & -1.23 & 1717 \\
\hline Ba II & 1 & 74 & 0.89 & & -1.24 & 1162 \\
\hline La II & & & -0.19 & & $<-1.41$ & 1544 \\
\hline Ce II & & & 0.02 & & $<-1.53$ & 1440 \\
\hline $\mathrm{Nd}$ II & 1 & 7 & -0.36 & & -1.86 & 1563 \\
\hline Sm II & & & -0.10 & & $<-1.10$ & 1560 \\
\hline
\end{tabular}

abundance in SX Cen is different than in RU Cen. Instead of a linear relation like in RU Cen, the relation is flatter for C, S, Zn and Na, which show solar abundances, and drops steeply only for elements with a condensation temperature higher than about $1200 \mathrm{~K}$ ranging from -0.8 for $[\mathrm{Mn} / \mathrm{H}]$ down to -2.0 for $[\mathrm{Sc} / \mathrm{H}]$ and $[\mathrm{Ti} / \mathrm{H}] . N$ shows a super-solar abundance and may indicate the CNO-burning products were dredged-up prior to the depletion process, while the $s$-process abundances indicate that there is no evidence for AGB nucleosynthethic product enhancement.

\section{Spectral monitoring}

During our spectral monitoring campaigns we obtain highresolution but low signal-to-noise spectra with the main objective to obtain radial velocity measurements. Strong emission lines like $\mathrm{H} \alpha$ can, however, also be studied on the extracted spectrum itself. Although we will continue this monitoring, we feel confident that for both objects important conclusions can already be drawn based on the data obtained so far.

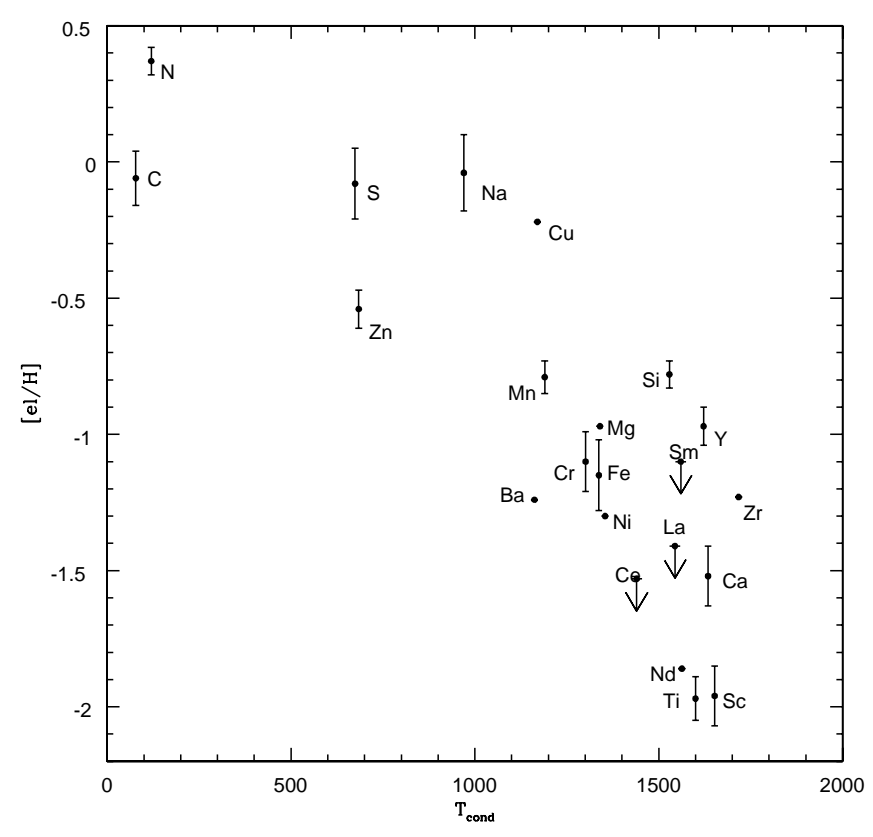

Fig. 8. The chemical deficiencies in the atmosphere of SX Cen against the dust condensation temperature of the chemical element.

The occurrence of line-profile deformation in the spectra of RV Tauri stars and the interpretation as being due to the passage of two shock waves in every pulsation cycle (period between two successive minima) was discussed for R Scuti and for AC Her by Gillet et al. (1989, 1990) and Lèbre \& Gillet (1991) and theoretically by Fokin (1994). The cross-correlation profile will, of course, also be affected by the unstable photosphere and the dynamics of the line-forming region are very well traced by the crosscorrelation profile.

\subsection{RU Cen}

\subsubsection{Radial velocities}

We can divide the observed correlation profiles roughly into three categories: Gaussian profiles, profiles showing line-deformation and profiles showing line-splitting. These different types of profiles are shown in Fig. 11.

At some photospheric phases it is therefore difficult to determine the radial velocity with a standard mask and we will construct special masks aiming at lines formed deep in the photosphere, in the near future for these pulsation phases. We have chosen till now the center of a Gaussian fit to be the radial velocity. In this way we obtained 48 good velocity measurements over a total time span of 407 days omitting one profile showing severe line-splitting with two equally strong components.

In Fig. 9 we have folded the radial velocity variations on the sampled cycles on the pulsation period of Pollard et al. (1996) refined by us to 64.64 days (see Sect. 5.1.2). We used JD 2448124.57 as the zero-point which is a phase of deep photometric minimum. Pollard et al. (1997) 


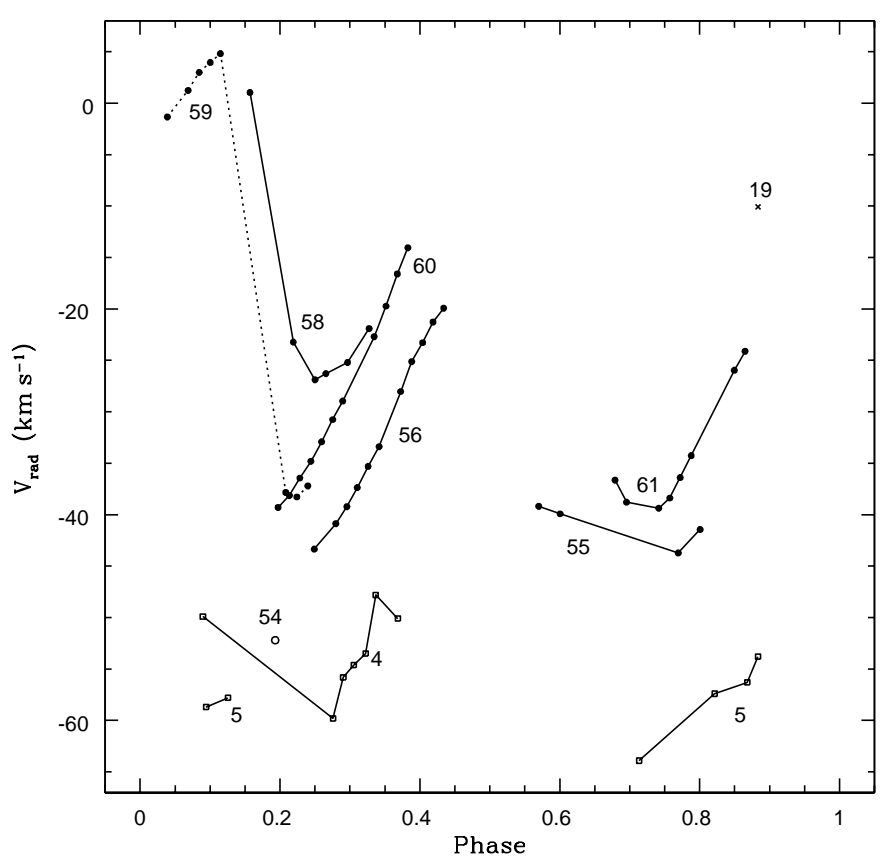

Fig. 9. The radial velocities versus photometric phase for RUCen. The used photometric period is 64.64 days with the zero-point of the pulsation cycle set to JD 2448124.57. CORALIE velocities are indicated with a full circle, the velocities of Pollard et al. (1997) with an open box, the velocity of the FEROS spectrum with an open circle and the cross indicates a CORAVEL measurment. Velocities of the same cycle are connected and each cycle is labeled. To prevent confusion the datapoints of cycle 59 are connected with a dotted line.

obtained data on two consecutive pulsation cycles and these data are included in the figure as well. They determined the velocity on the basis of one $\mathrm{C}$ absorption line at $6587.622 \AA$. Cycle 59, for which we have a good sampeling of both the maximum and the minimum, of our data shows that the amplitude of radial velocity variations due to pulsation can be as high as $45 \mathrm{~km} \mathrm{~s}^{-1}$. Although none of the photometric cycles is completely sampled, we obtained many data around phase 0.3 , which is around radial velocity minimum during a pulsation cycle. Around this phase we observe a cycle-to-cycle variability with a peak-to-peak amplitude of $\sim 30 \mathrm{~km} \mathrm{~s}^{-1}$. Since RU Cen is a very regular pulsator (Pollard et al. 1997), this large shift is difficult to interpret as due to pulsational irregularities. There is a large time gap between the radial velocities obtained by Pollard (cycles $4+5$ ) and the beginning of our monitoring (50 pulsation cycles) so the relative pulsation phase is not well constrained. However, there is clearly no overlap in radial velocities between our monitoring dataset (cycles 55 to 60 ) and the datapoints of Pollard et al.

Our homogeneous dataset alone, (cycles 54-61) we do have a clear systematic difference between the radial velocities obtained at different pulsation cycles: the radial velocities of different cycles are offset but show the same structure.
Both the peak-to-peak velocity difference at the same pulstation phase as well as the systematic offset between well sampled different pulsations cycles are significant. Since apart from binary motion, we do not know of any physical mechanism that can account for this behaviour, we conclude that RUCen is very likely a spectroscopic binary. More detailed and longer velocity monitoring is needed to make this statement conclusive and to obtain the orbital elements, but this will take several years more of regular monitoring.

\subsection{2. $\mathrm{H} \alpha$ line profile}

The $\mathrm{H} \alpha$ line-profile variations of RV Tauri stars are, in general, complex and at most photometric phases (strong) emission components are seen (e.g. Pollard et al. 1997).

RUCen is no exception and an example of a complex $\mathrm{H} \alpha$ line profile of RU Cen is shown in Fig. 4. To obtain a quantitative view of the changing strength of the $\mathrm{H} \alpha$ emission components, we adopt the same strategy as Pollard et al. (1997): we integrated the $\mathrm{H} \alpha$ line profile of RU Cen and folded the EW on the pulsation cycle. The EW is positive if the absorption is dominant, negative if the emission is dominant. After filtering of the cosmic hits and normalisation of the CORALIE spectral order, we computed the EW by direct integration. The uncertainty on the EW of the $\mathrm{H} \alpha$ line profile is mainly determined by the uncertainty on the continuum placement. We estimated the error to be around $15 \%$.

In Fig. 10 we confirm the results of Pollard et al. (1997) where the maximum of the $\mathrm{H} \alpha$ emission is found around pulsation phase 0.1 with a second lower maximum around phase 0.6. This is consistent with the passage of two shock waves during the full photometric period. For a period of 64.6 we found a small phase difference for the first peak so thanks to our long-term monitoring we could refine the period to 64.64 days, for which no shift of the maximum is observed. We have adopted this period throughout this article.

In RU Cen the primary shock is accompanied by linesplitting $(\varphi=0.07-0.26)$ or line-deformation $(\varphi=$ $0.16-0.30)$. The line-splitting points to a very strong shock. The perturbation of the line-forming region caused by the primary shock diminishes which can be seen in the gradually changing profile from line-deformation to Gaussian between phases 0.25 and 0.37 . For RU Cen the same is observed during the secondary shock with linedeformation $(\varphi=0.57-0.60)$ and even line-splitting $(\varphi=$ $0.68-0.76)$. Before the primary shock $(\varphi=0.77-0.04)$ the atmosphere is no longer perturbed and the profiles are again Gaussian (see Fig. 10).

\subsubsection{He-emission}

In RU Cen both shocks are so energetic that even He line emission is observed at these phases (see Fig. 12) which is, to our knowledge not yet reported in the literature. He line 


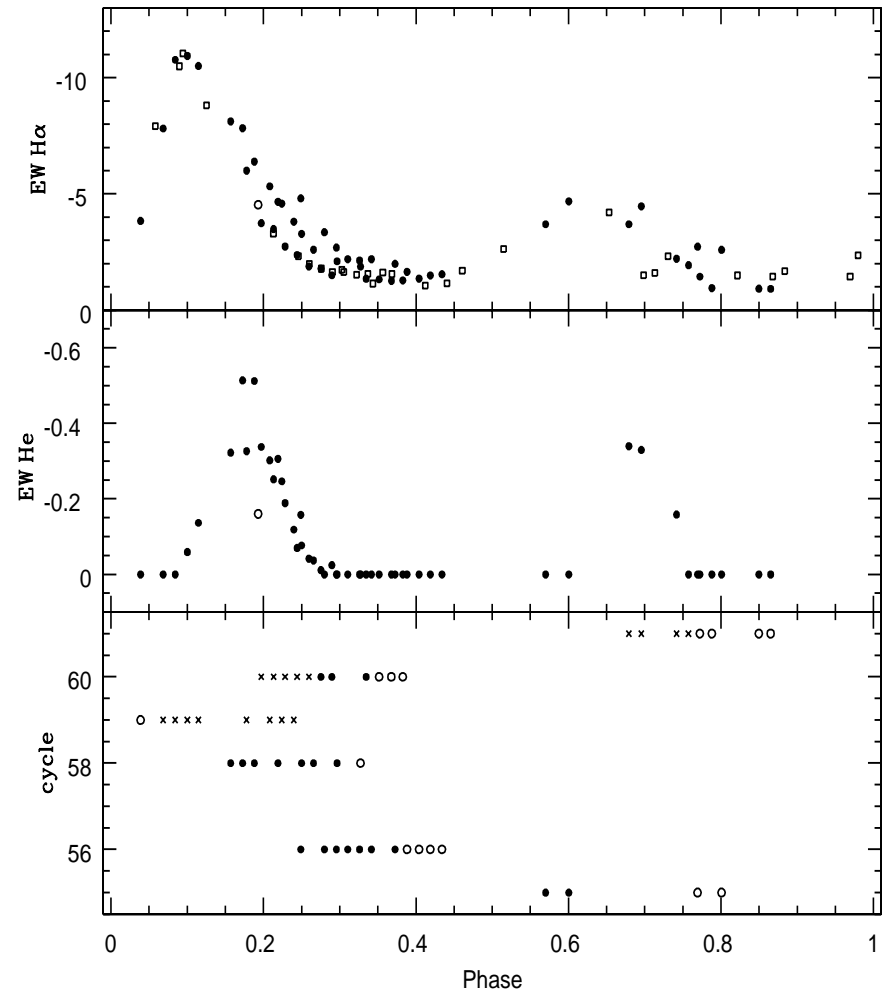

Fig. 10. Upper part: the equivalent width of the $\mathrm{H} \alpha$ line profiles versus photometric phase for RUCen. CORALIE data are indicated with a full circle, the data of Pollard with an open box and the result of the FEROS spectrum with an open circle. Middel part: the equivalent width of the He-line at $5876.50 \AA$, the result of the FEROS spectrum is indicated with an open circle. Lower part: line-splitting (crosses) occurs at $\varphi=0.07-0.26$ and $\varphi=0.68-0.76$; line-deformation (filled circles) at $\varphi=0.16-0.30$ and $\varphi=0.57-0.60$ and Gaussian profiles (open circles) are seen at $\varphi=0.33-0.43$ and at $\varphi=0.77-0.04$. The adopted photometric period is 64.64 days with the zero-point set to JD 2448124.57.

emission is not a general characteristic of RV Tau stars but there are a few individual stars for which it is also cummunicated in the literature: for $\mathrm{U}$ Mon this line emission is strong and present during a significant phase interval of the puslation cycle (Preston 1964), while for R Sct (Gillet et al. 1989) and AC Her (Baird 1982) He emission is only detected at a few spectra during monitoring campaigns.

Thanks to our good sampling, we can describe the $\mathrm{He}$ line emission behaviour during the pulsation cycle. We integrated the emission line of He at $5876.5 \AA$ and folded the EW of the line with the pulsation period. We present the result in the middle part of Fig. 10. Like for $\mathrm{H} \alpha$ the figure shows two maxima of the EW: a strong one around $\varphi=0.18$ and a weaker one around $\varphi=0.69$, corroborating the two-shock model. The maxima are well defined and narrower in phase-space than the $\mathrm{H} \alpha$ peaks shown in the upper part of the figure. The He emission is associated with strong shocks but there is a clear significant phase delay of 0.08-0.09 between the maxima of the $\mathrm{H} \alpha$ emission and those of He emission. Detailed modelling of the

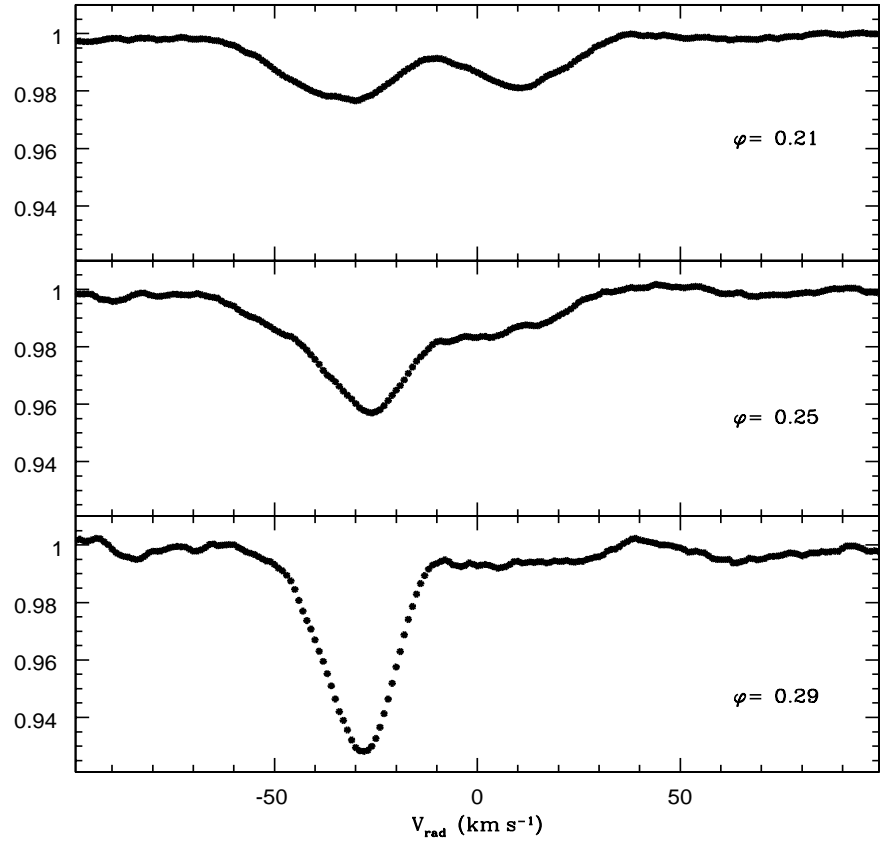

Fig. 11. Different types of cross-correlation profiles: linesplitting at $\varphi=0.21$, line-deformation at $\varphi=0.25$ and a Gaussian profile at $\varphi=0.29$.

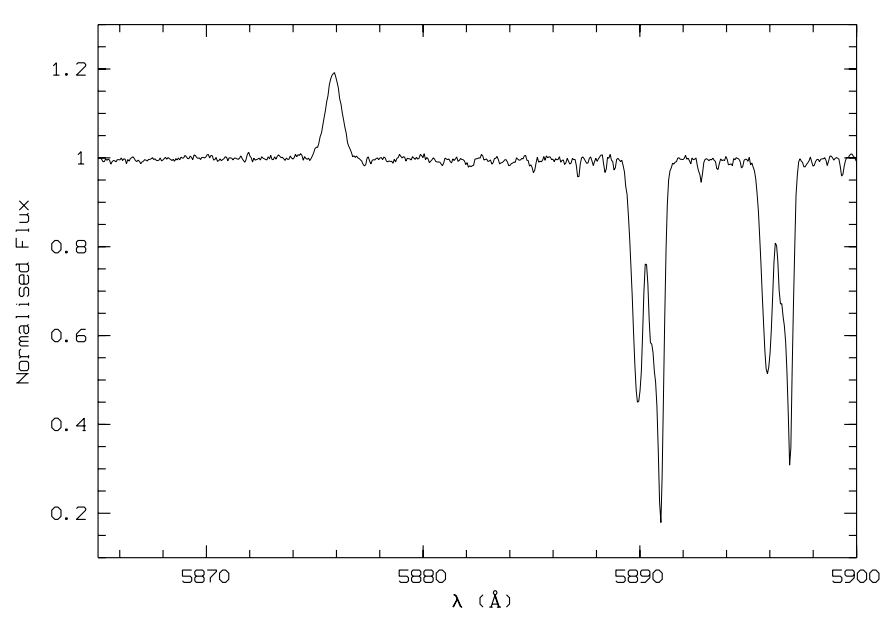

Fig. 12. The normalised FEROS spectrum of RU Cen around the He I emission line at $5876.5 \AA$.

behaviour of the outer atmospheric layers constrained by this type of monitoring is needed to understand the pulsational behaviour of RU Cen in particular and RV Tau stars in general but is beyond the scope of this paper.

\section{2. $S X C e n$}

\subsubsection{Radial velocities}

We determined the radial velocities for SXCen in the same way as for RUCen but we rejected three profiles: two because they were very wide, asymmetric and without a clear minimum, one because of strong line-splitting. As in RU Cen, three different types of correlation profiles 


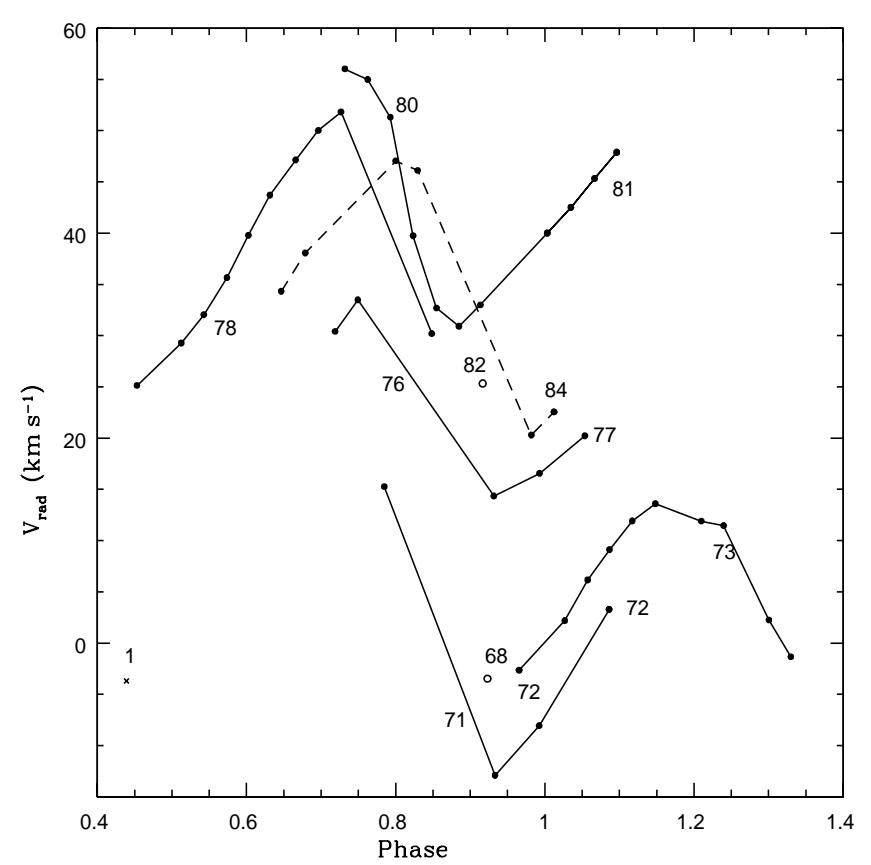

Fig. 13. CORALIE radial velocities versus photometric phase for SX Cen. The period used is 32.8642 days with zero point on JD 2447324.97. Velocities of the same cycle are connected. The velocity of the FEROS spectrum is indicated with an open circle while the cross indicates the CORAVEL velocity. We give the cycle number starting arbitrarily at 1 for the oldest datapoint. The measurements cluster in the 0.7-1.2 phase interval, so we show the phase-diagram in the $0.4-1.4$ phaserange.

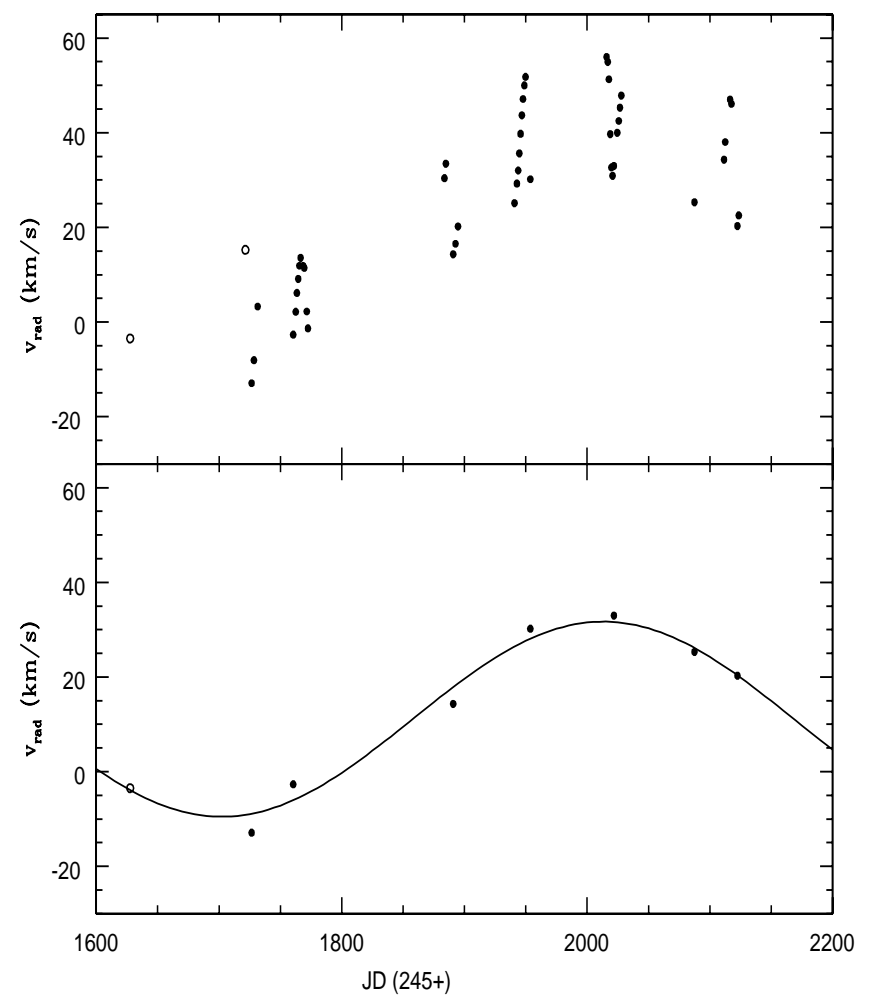

Fig. 14. The top panel shows all the radial velocities of SX Cen. The bottom panel only the data around phase 0.92 of cycles $1,4,5,9,13,15$ and 16 . The full line present a sine-fit with a period of 621 days. were observed: Gaussian profile, line-deformation and linesplitting (which was only observed once). These different types of profiles are shown in Fig. 15.

This resulted in 46 velocity measurements over a total time span of 402 days. Figure 13 shows the radial velocity versus the pulsation phase. Since many data points fall in the 0.7-1.2 phase interval, we have plotted a $0.4-$ 1.4 abscissa range. For the pulsation period we used $P=$ 32.8642 days (Kholopov et al. 1999) and the zero-point of the pulsation cycle was set to JD 2447324.97 (Shenton et al. 1994) which is the phase of deep photometric minimum.

Shenton et al. (1994) carried out multiwavelength observations of SX Cen and noted that SX Cen pulsates in a purely radial mode, its radius showing approximately sinusoidal variations. Unfortunately we do not have a complete well sampled pulsation cycle, but the radial velocity variations due to the pulsations have probably a lower amplitude and are more regular than for RU Cen. Furthermore our data show that SX Cen has two velocity cycles per pulsation period as is observed in many RV Tauri stars (Pollard et al. 1997).

The main point in Fig. 13 are the large cycle to cycle radial velocity differences at the same photospheric phases. The different cycles show a consistent picture in which it is clearly the mean radial velocity that changes. This is illustrated in the top panel of Fig. 14 where we see that the mean radial velocity of every run has a peak-topeak amplitude of some $40 \mathrm{~km} \mathrm{~s}^{-1}$. We interpret this as due to orbital motion of the object and classify SX Cen as a spectroscopic binary.

We plotted the measured radial velocities of different cycles around the best sampled photospheric phase (phase 0.92) in Fig. 14 bottom panel and computed a least square sine-fit through the data points. The period deduced was $621 \pm 31$ days with a system velocity of $11.0 \pm 0.1 \mathrm{~km} \mathrm{~s}^{-1}$ and an amplitude of $20.6 \pm 0.1 \mathrm{~km} \mathrm{~s}^{-1}$. This implies a mass function of $0.56 M_{\odot}$, a $\sin i=1.2 \mathrm{AU}$. Assuming a typical mass of $0.6 M_{\odot}$ for $M_{1}$, the minimal mass of the secondary would be $1.2 M_{\odot}$. As there is no evidence for a white dwarf companion of this mass, we assume the secondary to be an unevolved main sequence object. Since the sampling of the data is not optimal and we did not observe yet during a full orbit, a better knowledge of all the orbital elements will only become available after our planned intensive monitoring campaigns. Ideally we need to acquire several orbital cycles but, obviously, that will take several years.

\subsection{2. $\mathrm{H} \alpha$ line profile}

The $\mathrm{H} \alpha$ line profile of SX Cen is clearly different from that of RUCen since on every photometric phase the strong photospheric absorption is prominent. The strength of the emission components does vary (see Fig. 7) but our CORALIE data are of too low a signal-to-noise to perform the same exercise as for RU Cen. We could not detect He 


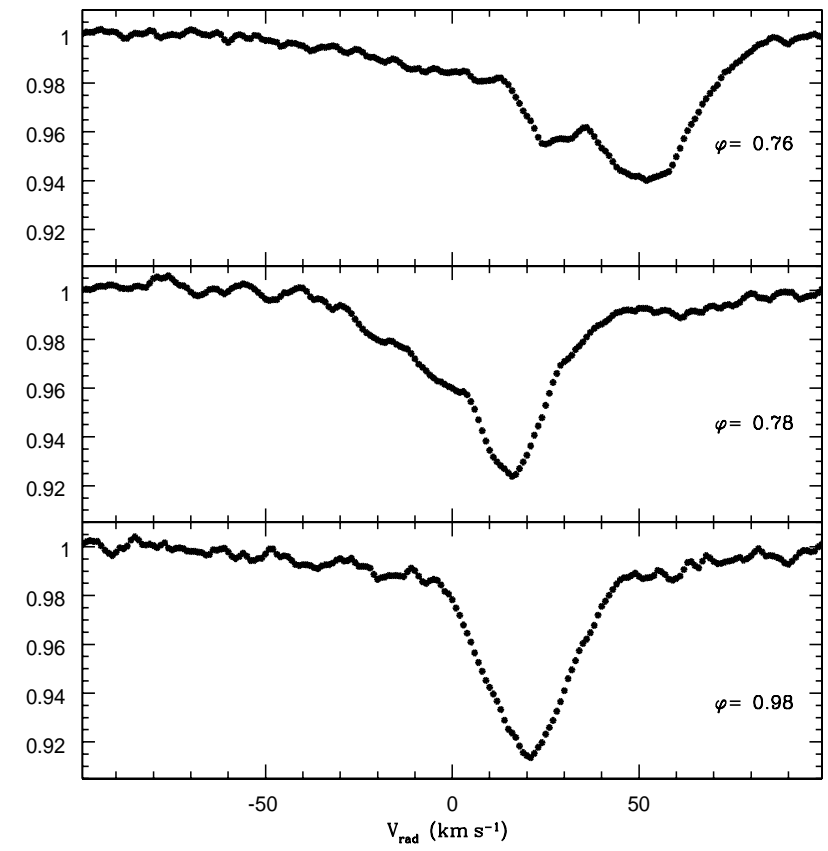

Fig. 15. Different types of cross-correlation profiles observed for SX Cen: (from bottom to top) a Gaussian profile, line distortion and line splitting.

line emission in any of the obtained spectra and we conclude that the shocks in SX Cen are probably weaker than in RU Cen.

\section{Discussion}

The main conclusions of our detailed chemical analysis and our long-term radial velocity monitoring with CORALIE are that: 1) the RV Tauri stars RUCen $([\mathrm{Fe} / \mathrm{H}]=-1.9 ;[\mathrm{Zn} / \mathrm{Fe}]=+0.9)$ and SX Cen $([\mathrm{Fe} / \mathrm{H}]=$ $-1.1 ;[\mathrm{Zn} / \mathrm{Fe}]=+0.6)$ are depleted in refractory elements; 2) both show strong evidence for binary motion such that we classify both as spectroscopic binaries; 3) the SED of both stars indicate the presence of large amounts of hot dust; 4) the spectral monitoring of RUCen is consistent with the picture described previously in which there is evidence for the passage of two shock waves in every pulsation cycle. Both shocks are energetic enough to excite He in the case of RU Cen and, last but not least, 5) for SX Cen the deduced orbital period of $621 \pm 31$ days is, within the error, the same as the period of the long-term variability of the mean light (RV Tauri photometric b phenomenon).

The depletion of refractory elements in many RV Tauri stars is now well documented (see Giridhar et al. 2000 and references therein) and since all the Preston B class objects studied till now are strongly affected, the depletion of RU Cen and SX Cen discussed here is not surprising. In the picture described by Waters et al. (1992), the basic structure for the depletion process to be effective for evolved stars is the presence of a stable circumstellar environment where the timescale and expected densities are favorable for gas-dust separation and gas accretion. The binary nature of many RV Tauri stars, as proposed by Van Winckel et al. (1999), is, however, not generally accepted. Based on the propositions of Mathis \& Lamers (1992), Giridhar et al. (2000) sketch also a single star depletion process. In such a process it is, however, not clear how the special characteristics of the dusty circumstellar environment (see introduction) around RV Tauri stars are produced and maintained.

Van Winckel et al. (1999) argued that the basic structure of many RV Tauri stars (certainly the depleted ones) is a binary with a stable circumstellar dusty disc. The evolution and evolutionary timescale of the IR-excess of RV Tau stars is therefore not determined by the outflow velocity of the detaching dust shell, but by dust processing in the radiative environment of the central stars. The stability of the disc-structure is well documented, not only in RV Tauri stars, but also in post-AGB binaries and even some AGB objects (see introduction).

Giridhar et al. (2000) show that there is no expected correlation between the strength of the IR-excess and the severeness of the depletion observed in RV Tauri stars. Also for the strongly depleted post-AGB binaries there is no such correlation: the stars display a bewildering variety of IR excesses but they are all strongly depleted. The orbits found in these binaries prevented them from evolving along and off the AGB on single-star evolutionary tracks. Moreover, in several of them the disc is resolved and proven to be very stable, while some do not even have a (detected) IR-excess. The variety of IR excesses observed in these strongly depleted stars is also determined by a difference in the level of dust processing and disc evolution.

In the prototypical RV Tauri star ACHer this basic structure is spatially resolved: the star shows clear chemical depletion patterns (Van Winckel et al. 1998; Giridhar et al. 2000) and detailed radial velocity monitoring revealed it is a binary with an orbital period of about 1200 days (Van Winckel et al. 1998). The observational evidence for the presence of a stable strongly processed dusty disc comes from mid-IR spectra indicating the presence of highly crystalline oxygen rich material (Molster et al. 1999), narrow and faint CO microwave emission (Bujarrabal et al. 1988; Jura \& Kahane 1999) and the presence of large grains (Shenton et al. 1995). Recently the circumstellar material was resolved into two spots at mid-IR wavelength which is interpreted as due to a resolved dusty ring (Jura et al. 2000).

The binary nature of RUCen and SXCen together with the characteristics of their SED, indicate that this picture can also apply for these stars. Moreover, the observational characteristic that the RV Tauri stars occupy a special region in the IRAS colour-colour diagram in combination with an often observed K and L excess (see e.g. Lloyd Evans 1999) can be naturally explained since the dust close to the central stars will remain hot, which would not be the case in a slowly expanding shell. The existence of a clear period-luminosity relation for type II Cepheids with the RV Tauri stars occupying the luminous end of the instability strip (Alcock et al. 1998) suggest that not the 
pulsation itself is induced/changed by binary interaction but that it is the location in the HR-diagram and/or the evolutionary timescale which is strongly affected by the presence of a binary companion.

SX Cen is an RV Tauri star of photometric class b with a secular photometric variation of 615 days (Lloyd Evans 1985) and turns out to be a binary with an orbital period of very similar length. The same was observed in the strongly depleted RV Tau star HD 52961 (Van Winckel et al. 1999).

The idea that the photometric $b$ phenomenon itself is linked with binarity is not new (e.g. Lloyd-Evans 1985; Fokin 1994). Since the RVb stars have in general redder colours than the RVa stars (Lloyd Evans 1985) variable circumstellar absorption in the variable line of sight during orbital motion is a very good candidate to explain the RVb phenomenon (Percy 1993; Waelkens \& Waters 1993; Fokin 1994). Note that not all the characteristics of the light curve of some objects can be explained by variable circumstellar absorption alone: in U Mon for instance, amplitude damping is detected during the long-term photometric minimum (Pollard et al. 1996) which is difficult to account for in a simple geometric picture. ACHer is a binary of photometric class "a" while SX Cen is a binary of photometric class "b". Nevertheless, in this paper we have good evidence that both stars have intrinsically the same structure. This confirms the suggestion that the photometric class of RV Tauri stars does not represent a physical difference but merely a geometric projection effect (Van Winckel et al. 1999). SX Cen would then be an object with a high binary inclination (near edge-on) and the long term photometric variation would be mainly due to the variable circumstellar extinction.

Although the RV Tauri class of objects is rather inhomogeneous and it is dangerous to overgeneralise results on a limited number of objects to a whole class of stars, the binary nature of RU Cen and SX Cen strengthens the statement that binarity in combination with the presence of a stable circumstellar dusty disc, is a widespread phenomenon among RV Tauri stars. Although many observational characteristics of RV Tauri stars can be explained in this picture, only long-term multi-wavelength spectral and photometric monitoring will be able to study the enigmatic objects in detail. Needless to say this is not a trivial observational challenge. Moreover, the spe(c)(t)ial characteristics of the circumstellar environment should certainly not be overlooked in these studies. The current and upcoming high-spatial resolution IR imaging facilities will be very valuable, also in this respect.

Acknowledgements. The authors thank the staff of the Geneva observatory for the allocated time on the $1.2 \mathrm{~m}$ Euler telescope and for the use of the reduction software package for the CORALIE spectra as well as for the generous award of telescope time on the photometric telescope. The staff of the Instituut voor Sterrenkunde are acknowledged for the assistance with the monitoring programmes. This research has made use of the SIMBAD database, operated at CDS, Strasbourg, France. TM acknowledge finacial support from the
Fund for Scientific Research of Flanders (FWO) under FKFO project number G.0265.97. HVW acknowledges financial support from the FWO as post-doc.

\section{References}

Alcock, C., Allsman, R. A., Alves, D. R., et al. 1998, AJ, 115, 1921

Anders, E., \& Grevesse, N. 1989, Geochimica et Cosmochimica Acta, 53, 197

Baird, S. R. 1982, PASP, 94, 850

Baranne, A., Queloz, D., \& Mayor, M., et al. 1996, A\&AS, 119,373

Bond, H. E., \& Luck, R. E. 1987, ApJ, 312, 203

Bond, H. E., Fullton, L. K., Schaefer, K., et al. 1997, IAU Symp., 180, 21

Bujarrabal, V., Bachiller, R., Alcolea, J., \& Martín-Pintado 1988, A\&A, 206, L17

Gehrz, R. D. 1972, ApJ, 178, 715

Gehrz, R. D., Ney, E. P. 1972, PASP, 84, 768

Gillet, D., Burki, G., \& Duquennoy, A. 1990, A\&A, 237, 159

Gillet, D., Duquennoy, A., Bouchet, P., \& Gouiffes, C. 1989, A\&A, 215, 316

Giridhar, S., Rao, N. K., \& Lambert, D. L. 1994, ApJ, 437, 476

Giridhar, S., Lambert, D. L., \& Gonzalez, G. 1998, ApJ, 509, 366

Giridhar, S., Lambert, D. L., \& Gonzalez, G. 2000, ApJ, 531, 521

Gonzalez, G., Lambert, D. L., \& Giridhar, S. 1997a, ApJ, 479, 427

Gonzalez, G., Lambert, D. L., \& Giridhar, S. 1997b, ApJ, 481, 481

Fokin, A. B. 1994, A\&A, 292, 133

Jura, M. 1986, ApJ, 309, 732

Jura, M., \& Kahane, C. 1999, AJ, 521, 302

Jura, M., Chen, C., \& Werner, M. W. 2000, ApJ, 541, 264

Kodaira, K., Greenstein, J. L., \& Oke, J. B. 1970, ApJ, 159, 485

Kholopov, P. N., Samus, N. N., Frolov, M. S., et al. 1999, Combined General Catalogue of Variable Stars, VisieR online data Catalog, II/214A (originally published in Moscow: Nauka Publishing House (1985-1988))

Kupka, F., Piskunov, N., Ryabchikova, T. A., Stempels, H. C., \& Weiss, W. W. 1999, A\&AS, 138, 119

Lambert, D. L., Hinkle, K. H., \& Luck, R. E. 1988, ApJ, 333, 917

Lloyd Evans, T. 1985, MNRAS, 217, 493

Lloyd Evans, T. 1999, IAUS, 191, 459

Lodders, K., \& Fegley, B. 1988, The Planetary Scientist's Companion (Oxford Univ. Press), 83

Luck, R. E., \& Bond, H. E. 1989, ApJ, 342, 476

Mathis, J. S., \& Lamers, H. J. G. L. M. 1992, A\&A, 259, L39

Molster, F. J., Waters, L. B. F. M., Trams, N. R., et al. 1999, A\&A, 350, 163

O'Connell, D. J. K. 1933, Harvard Bull., 893, 14

Percy, J. R. 1993, in Luminous High-Latitude Stars, ed. D. D. Sasselov, 295

Pollard, K. R., \& Cottrell, P. L. 1995, ASP Ser. 83, ed. R. S. Stobie, \& P. A. Whitelock, 409

Pollard, K. R., Cottrell, P. L., Kilmartin, P. M., \& Gilmore, A. C. 1996, MNRAS, 279, 949 
Pollard, K. R., Cottrell, P. L., Lawson, W. A., Albrow, M. D., \& Tobin, W. 1997, MNRAS, 286, 1

Preston, G. W. 1964, ApJ, 140, 173

Preston, G. W., Krzeminski, W., Smak, J., \& Williams, J. A. 1963, ApJ, 137, 401

Queloz, D., Casse, M., \& Mayor, M. 1999, in IAU Colloq., 170, Precise radial velocities, ed. J. B. Hearnshaw, \& C. D. Scarfe (Victoria BC Canada), ASP Conf. Ser., 13

Roddier, F., Roddier, C., Graves, J. E., \& Northcott, M. J. 1995, ApJ, 443, 249

Shenton, M., Evans, A., Albinson, J. S., et al. 1994, A\&A, 292, 102

Shenton, M., Evans, A., \& Williams, P. M. 1995, MNRAS, 273, 906

Van Winckel, H. 2001, Ap\&SS, 275, 159

Van Winckel, H., \& Reyniers, M. 2000, A\&A, 354, 135

Van Winckel, H., Waelkens, C., \& Waters, L. B. F. M. 1995, A\&A, 293, L25
Van Winckel, H., Waelkens, C., Waters, L. B. F. M., et al. 1998, A\&A, 336, 263

Van Winckel, H., Waelkens, C., Fernie, J. D., \& Waters, L. B. F. M. 1999, A\&A, 343, 202

Voûte, J. 1940, Lembang Ann., 8, 42

Waelkens, C., \& Waters, L. B. F. M. 1993, ASP Conf. Ser., 45, 219

Waelkens, C., Lamers, H. J. G. L. M., Waters, L. B. F. M., et al. 1991a, A\&A, 242, 433

Waelkens, C., Van Winckel, H., Bogaert, E., \& Trams, N. R. 1991b, A\&A, 251, 495

Waelkens, C., Van Winckel, H., Trams, N. R., \& Waters, L. B. F. M. 1992, A\&A, 256, L15

Waters, L. B. F. M., Trams, N. R., \& Waelkens, C. 1992, A\&A, 262, L37

Waters, L. B. F. M., Waelkens, C., \& Van Winckel, H. 1997, IAUS, 180, 313 